\title{
Tomografia computadorizada do encéfalo do cão: aspectos da normalidade e correlação anatômica
}

\author{
[Computed tomography of the dog's brain: normal aspects and anatomical correlation] \\ C.A.B. Lorigados ${ }^{1}$, A.C.B.F. Pinto ${ }^{2}$ \\ ${ }^{1}$ Faculdades Metropolitanas Unidas (UniFMU) - São Paulo, SP \\ ${ }^{2}$ Faculdade de Medicina Veterinária e Zootecnia - Universidade de São Paulo (USP) - São Paulo, SP
}

\section{RESUMO}

Obtiveram-se imagens tomográficas sem alterações da cabeça de cães, visando à familiarização com os aspectos normais do encéfalo e correlacionaram-se os achados com a respectiva anatomia da região estudada. Várias estruturas anatômicas foram identificadas, tais como o parênquima dos lobos frontal, parietal, temporal e occipital, a fissura longitudinal, o sistema ventricular, o cerebelo, o bulbo olfatório, o corpo caloso, o diencéfalo, a ponte, a medula oblonga e o sulco quiasmático, de forma direta ou relacionando-as com estruturas vizinhas que auxiliaram na sua identificação.

Palavras-chave: cães, encéfalo, anatomia, tomografia computadorizada

\begin{abstract}
Normal tomographic images of dog's heads were obtained, aimed to familiarize them with the normal aspects of the brain and correlate these findings with the relevant anatomy of the region studied. Several anatomical structures, such as the parenchyma of the frontal, parietal, temporal and occipital lobes, the longitudinal fissure, the ventricular system, the cerebellum, the olfactory bulb, the corpus callosum, diencephalon, the pons, the medulla oblongata and the chiasmatic sulcus were directly identified or were related to neighboring structures which helped in their identification.
\end{abstract}

Keywords: dogs, brain, anatomy, computed tomography

\section{INTRODUÇÃO}

O estudo do sistema nervoso central é uma das áreas de maior interesse na medicina e vem crescendo na medicina veterinária à medida que novas modalidades diagnósticas por imagem têm se tornado uma realidade neste meio. O campo do diagnóstico por imagem na neurologia sofreu um grande avanço tecnológico, com o surgimento da tomografia computadorizada (TC) e da ressonância magnética (RM).

A ressonância magnética tem sido considerada o padrão ouro na avaliação do encéfalo, contudo o alto custo do equipamento e de sua manutenção tem limitado sua utilização. Graças às características da TC de também obter imagens seccionais, sem sobreposição de estruturas, além

Recebido em 11 de novembro de 2011

Aceito em 29 de novembro de 2012

E-mail: clorigados@usp.br de apresentar uma boa diferenciação entre os tecidos moles, essa modalidade de diagnóstico por imagem foi adotada amplamente para tal finalidade (Hathcock e Stickle, 1993; Tidwell e Jones, 1999). Os primeiros estudos tomográficos em pequenos animais surgiram na década de 80 (Fike et al., 1980; Fike et al., 1981; Loden et al., 1983). A TC é capaz de fornecer inúmeras informações a respeito das afecções que podem acometer o encéfalo, tais como a localização, as dimensões, sua natureza intra ou extra-axial, a densidade, o grau de realce após a administração de contraste iodado, além de mostrar o deslocamento ou a deformidade de estruturas anatômicas que possam ser provocados por essas lesões (efeito de massa), áreas focais de calcificação, hemorragia, edema e dilatação do sistema ventricular (Jeffery et al., 1992; Tucker e Gavin, 1996; Thomas, 1999; Snyder et al., 2006; Mackillop et al., 2007; Dennler et al., 2007). 
A TC está se tornando uma modalidade diagnóstica cada vez mais acessível no Brasil. A avaliação de alterações cranianas é uma das maiores indicações do exame tomográfico, quando não se tem disponível a ressonância magnética, tornando-se importante a familiarização com os aspectos da normalidade da região em estudo. Além disso, assim como ocorrem artefatos em outras modalidades de imagem, eles também são gerados nas imagens tomográficas do crânio, como streaks e endurecimento do feixe (Hathcock e Stickle, 1993; Tidwell e Jones, 1999). Conhecer esses aspectos é fundamental para evitar interpretações equivocadas.

O objetivo deste trabalho foi apresentar imagens tomográficas do encéfalo de cães sem alterações e correlacioná-las com a anatomia macroscópica da região, a fim de servir de embasamento para sua utilização na rotina clínica.

\section{MATERIAL E MÉTODO}

Foram utilizados 10 cães adultos, sem raça definida, seis machos e quatro fêmeas, com peso entre 15 e $20 \mathrm{~kg}$, provenientes do Centro de Controle de Zoonoses de São Paulo, recémsacrificados. Quanto à conformação craniana, os cães apresentavam classificação fenotípica mesaticefálica.

Os exames tomográficos da cabeça foram obtidos em um equipamento CT-MAX 640 (General Eletric), de terceira geração. As imagens foram fotografadas em câmera multiformato MFC640, em filme tamanho $35 \times 43 \mathrm{~cm}$ (Kodak Brasileira Com. Ind. Ltda), o qual foi posteriormente revelado e fixado em processadora automática RPX-OMAT Processor (Easteman Kodak Company). Para a obtenção dos cortes transversais, os animais foram posicionados em decúbito esternal com os membros torácicos tracionados caudalmente. A partir de uma radiografia digital dorsal da cabeça (scout), os cortes transversais foram obtidos com orientação rostrocaudal, iniciando-se na região do bulbo olfatório e estendendo-se até a região do forame magno.

Para a obtenção dos cortes dorsais, os cães foram posicionados em decúbito dorsal, com o auxílio de um colchão de espuma em formato de calha, e os membros torácicos tracionados caudalmente.
A cabeça foi posicionada formando um ângulo aproximado de $90^{\circ}$ com a coluna cervical. A partir de uma radiografia digital lateral (scout), os cortes dorsais foram obtidos com orientação ventrodorsal, iniciando-se próximo à região palatina e estendendo-se até a porção mais dorsal da cabeça. A espessura dos cortes transversais e dorsais foi de $5 \mathrm{~mm}$, com incremento de $5 \mathrm{~mm}$ entre eles. O ajuste da técnica foi de $120 \mathrm{kV}, 55 \mathrm{a}$ $77 \mathrm{~mA}$ e três segundos de tempo de aquisição para cada corte realizado. Foi utilizada matriz de 640 X 640 pixels. Todos os animais tiveram as cabeças congeladas, e com o auxílio de uma serra elétrica em fita, foram seccionados nas marcações realizadas, cinco em cortes dorsais, cinco em cortes transversais.

Os encéfalos foram analisados somente macroscopicamente, não se evidenciando alterações perceptíveis nas regiões em que os cortes foram realizados. Com base neste banco de imagens, foi estudada a correlação das imagens tomográficas e do encéfalo com a anatomia macroscópica, com auxílio de livros de anatomia (Beitz e Fletcher, 1999; Schaller, 1999; Dyce e Sack, 2004; König et al., 2004), de diagnóstico por imagem veterinário (Feeney et al., 1991; Assheuer e Sager, 1997) e dados de uma tese de doutorado sobre ressonância magnética da cabeça de cão (Hage, 2005).

\section{RESULTADOS}

As estruturas encefálicas identificadas nas imagens tomográficas foram: o parênquima dos lobos frontal, parietal, temporal e occipital, a fissura longitudinal, o sistema ventricular, o cerebelo, o bulbo olfatório, o corpo caloso, o diencéfalo, a ponte, a medula oblonga, o sulco quiasmático (Fig. 1-6). Outras estruturas anatômicas da cabeça foram identificadas para auxiliar a interpretação das imagens tomográficas: fissura orbitária, forame oval, forame alar, seio frontal, seio esfenoidal, nasofaringe, ramo da mandíbula, arco zigomático, osso pterigoide, bolha timpânica, meato acústico externo, além dos ossos que compõem a cavidade craniana (Fig. 1-6).

Os artefatos de imagem observados nos exames tomográficos da cabeça foram o endurecimento do feixe visto na região da fossa caudal do crânio e os streaks (Fig. 3C). 

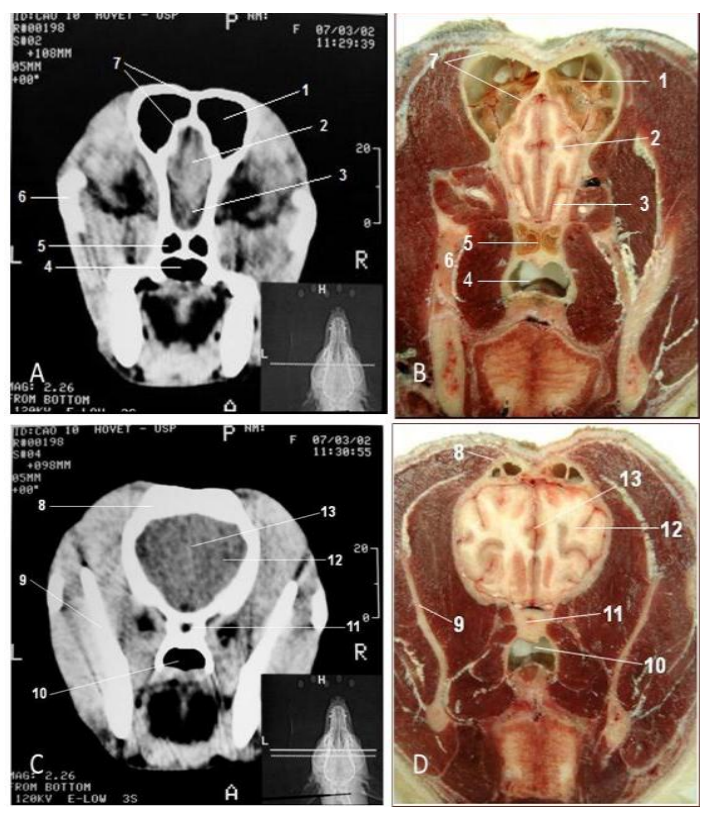

Figura 1. Imagens tomográficas em plano transversal da cabeça do cão (A e C) e cortes anatômicos correspondentes (B e D). 1- seio frontal; 2 e 12- lobo frontal; 3- bulbo olfatório; 4 e 10-nasofaringe; 5 e 11- seio esfenoidal; 6-arco zigomático; 7 e 8- osso frontal; 9- ramo da mandíbula; 13 fissura longitudinal. A linha pontilhada no scout no canto inferior direito das imagens tomográficas indica a topografia do corte.

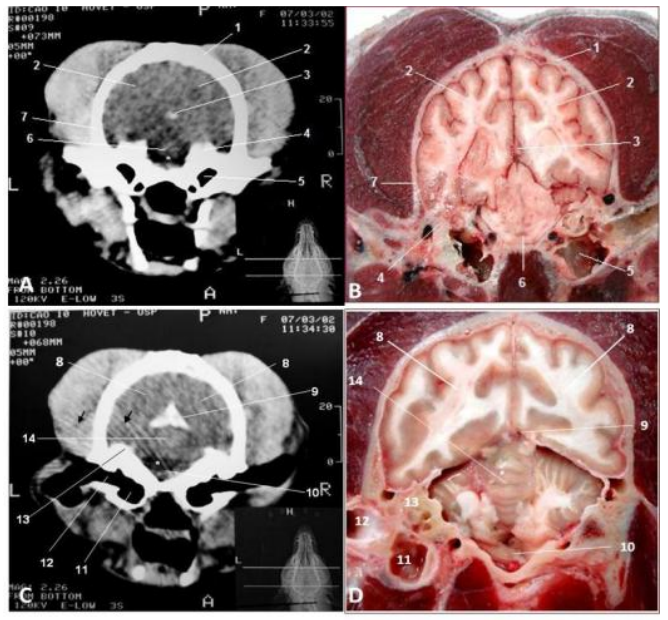

Figura 3. Imagens tomográficas em plano transversal da cabeça do cão (A e C) e cortes anatômicos correspondentes (B e D). 1-osso parietal; 2-lobo parietal; 3-tentório ósseo do cerebelo; 4-parte petrosa do temporal; 5-bolha timpânica; 6ponte; 7-parte escamosa do temporal; 8-lobo occipital; 9tentório ósseo do cerebelo; 10-medula oblonga; 11-bolha timpânica; 12-meato acústico externo; 13-parte petrosa do temporal; 14-cerebelo. A linha pontilhada no scout no canto inferior direito das imagens tomográficas indica a topografia do corte. Na figura $\mathrm{C}$, as setas indicam artefato streaks, e o asterisco artefato de endurecimento do feixe.

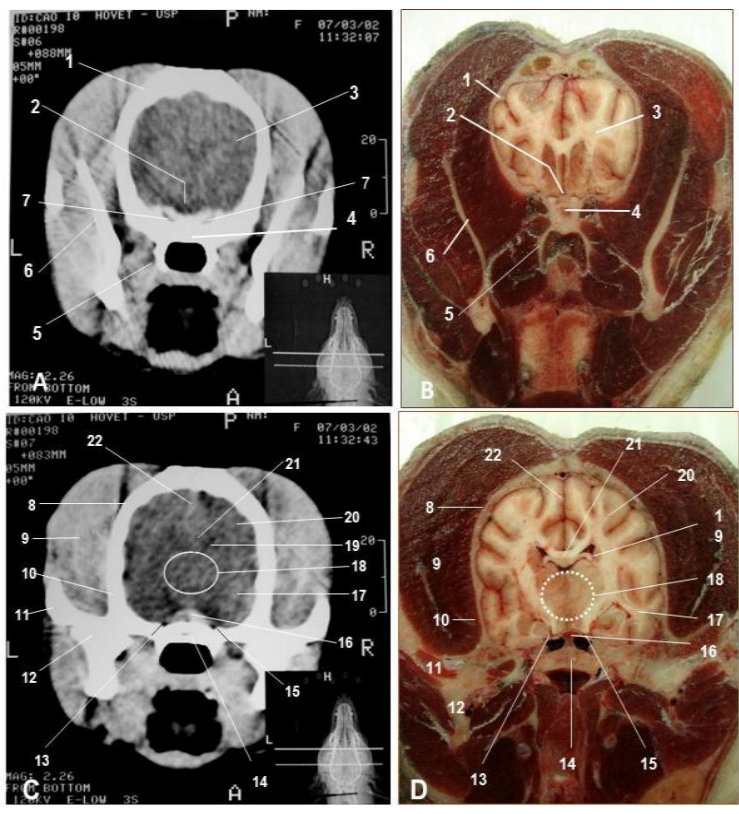

Figura 2. Imagens tomográficas em plano transversal da cabeça do cão $(\mathrm{A}$ e $\mathrm{C})$ e cortes anatômicos correspondentes (B e D). 1-osso frontal; 2-sulco quiasmático; 3-lobo frontal; 4-osso basisfenoide; 5osso pterigoide; 6-ramo da mandíbula; 7-fissura orbitária; 8- osso parietal; 9-músculo temporal; 10-osso temporal; 11-processo zigomático do osso temporal; 12-processo condilar da mandíbula; 13 e 15- forame oval; 14-osso basisfenoide; 16- dorso da sela; 17lobo temporal; 18- diencéfalo; 19-ventrículo lateral; 20-lobo parietal; 21- corpo caloso; 22-fissura longitudinal. A linha pontilhada no scout no canto inferior direito das imagens tomográficas indica a topografia do corte.

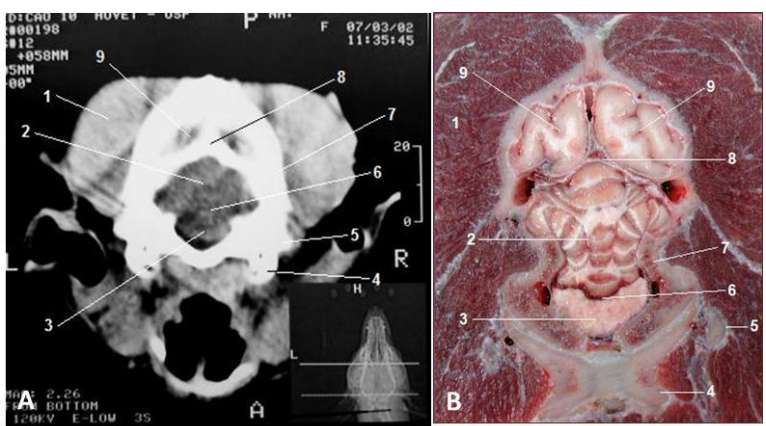

Figura 4. Imagem tomográfica em plano transversal da cabeça do cão (A) e corte anatômico correspondente (B). 1-músculo temporal; 2-cerebelo; 3-medula oblonga; 4-processo jugular do occipital; 5-processo mastoide da porção petrosa do temporal; 6quarto ventrículo, 7-osso occipital; 8-tentório ósseo do cerebelo; 9lobo occipital. A linha pontilhada no scout no canto inferior direito da imagem tomográfica indica a topografia do corte. 


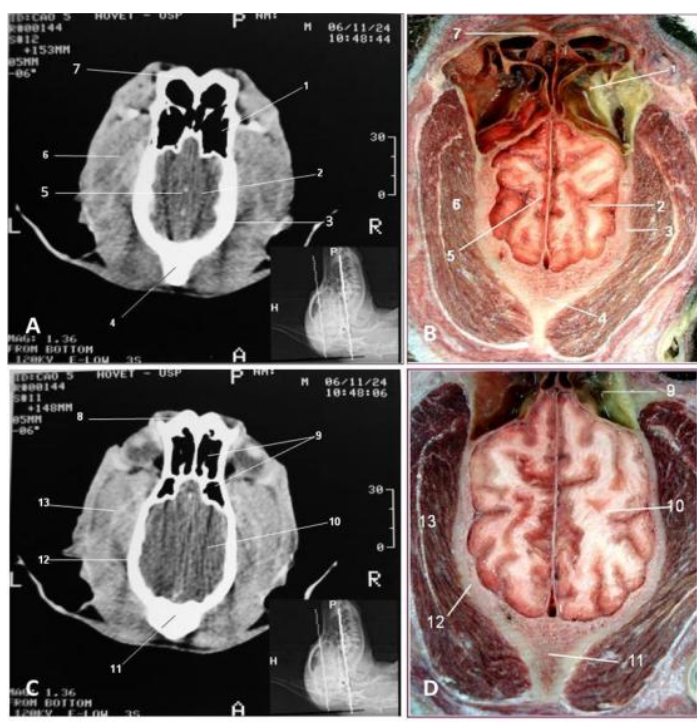

Figura 5. Imagens tomográficas em plano dorsal da cabeça do cão $(\mathrm{A}$ e C $)$ e cortes anatômicos correspondentes (B e D). 1seio frontal; 2- lobo parietal; 3-osso parietal; 4-osso occipital; 5-fissura longitudinal; 6-músculo temporal; 7 e 8-osso frontal; 9-seio frontal; 10-lobo parietal; 11-osso occipital; 12-osso parietal; 13-músculo temporal. A linha pontilhada no scout no canto inferior direito da imagem tomográfica indica a topografia do corte.

\section{DISCUSSÃO}

Apesar de a tomografia ser uma modalidade diagnóstica capaz de obter imagens com boa diferenciação entre os tecidos moles (Tucker e Gavin, 1996), várias estruturas encefálicas não apresentam características próprias na imagem capaz de diferenciá-las de outras estruturas adjacentes, tais como o corpo caloso, o diencéfalo (Fig. 2C), o cerebelo (Fig. 3C, 4A) e o quiasma óptico (Fig. 2A). Dessa forma, elas foram identificadas a partir de outras estruturas anatômicas que serviram como referência para sua localização. Estruturas ósseas cranianas e alguns forames, melhor observados em imagens com seleção de janela e nível adequados para avaliação óssea, também representam pontos referenciais importantes. Alguns estudos por ressonância magnética têm demonstrado a localização dos nervos cranianos, não sendo possível essa identificação por tomografia computadorizada, mas alguns forames podem indicar a região de sua emergência (Couturier et al., 2005). A fissura orbitária (Fig. 2A) é a região de emergência de vários nervos cranianos (III, IV, VI e o ramo maxilar do nervo trigêmeo). O

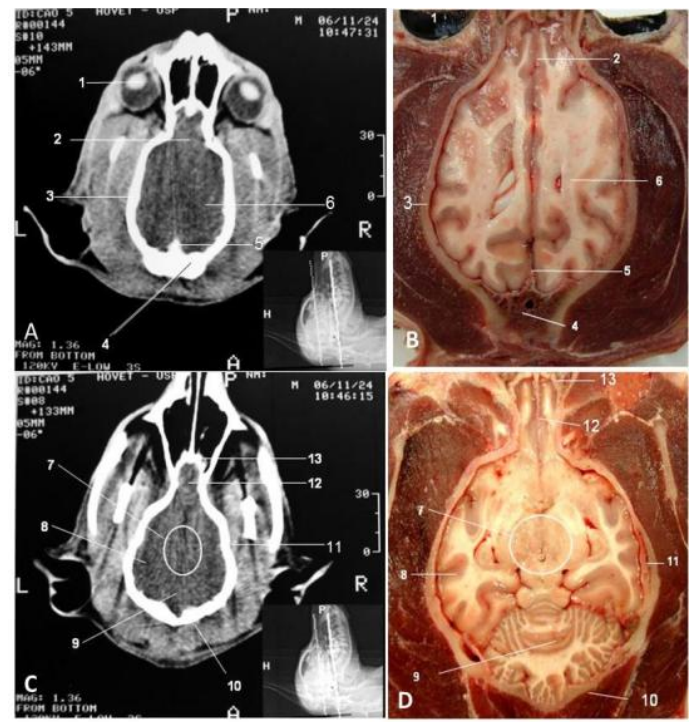

Figura 6. Imagens tomográficas em plano dorsal da cabeça do cão (A e C) e cortes anatômicos correspondentes (B e D). 1globo ocular; 2-lobo frontal; 3-osso parietal; 4-osso occipital; 5-tentório ósseo do cerebelo; 6-lobo parietal; 7-diencéfalo; 8lobo temporal; 9-cerebelo; 10-osso occipital; 11-osso temporal; 12-bulbo olfatório; 13-lâmina cribriforme. Imagens tomográficas em plano dorsal da cabeça do cão (A e C) e os cortes anatômicos correspondentes (B e D). A linha pontilhada no scout no canto inferior direito da imagem tomográfica indica a topografia do corte.

ramo mandibular do trigêmeo emerge através do forame oval (Fig. 2C). O tentório ósseo do cerebelo, bem identificado nas imagens (Fig. 4A), é um marco para a localização do cerebelo, o dorso da sela túrcica (Fig. 2C) indica uma localização imediatamente caudal à fossa hipofisária e à hipófise, e a fissura orbitária indica proximidade ao sulco quiasmático. Sabese que a diferenciação entre os tecidos na tomografia se deve a sua capacidade de atenuação dos raios X (Phelps et al., 1975) e que, em condições normais, os valores de atenuação dos raios $\mathrm{X}$ podem não ser significativamente diferentes entre determinadas estruturas, a exemplo do tecido cerebral e da região do diencéfalo. Mesmo os ventrículos laterais, preenchidos por liquor, em condições normais não são tão evidentes (Fig. 2D). Embora tenham sido identificados nos cortes transversais como estruturas hipoatenuantes, nos cortes dorsais a sua visibilização direta não foi observada nos cães estudados. Hage (2005), em sua tese, observou aspecto semelhante nas imagens por ressonância magnética, modalidade de imagem superior na identificação anatômica das estruturas encefálicas. $\mathrm{O}$ formato mais alongado 
e delgado do ventrículo obtido nesse plano de secção torna sua observação mais difícil. Contudo, quando ocorre a dilatação do sistema ventricular, sua identificação se torna fácil, sendo assim a TC um excelente método para esse diagnóstico. Os sulcos e os giros cerebrais, que podem ser identificados nas imagens por ressonância magnética (Hage, 2005), não são passíveis de identificação pela tomografia computadorizada, tampouco a diferença entre a substância branca e a substância cinza, tão bem mostrada nas imagens tomográficas do homem. Embora equipamentos mais modernos sejam capazes de oferecer imagens com maior resolução, acredita-se que este não seja o fator limitante, pois as imagens observadas nos trabalhos da literatura, algumas realizadas em equipamentos superiores ao utilizado neste experimento, também não forneceram tal diferenciação (Snyder et al., 2006; MacKillop et al., 2007; Dennler et al., 2007).

Quanto aos artefatos de imagem observados, streaks são linhas paralelas que geralmente ocorrem adjacentes a estruturas de alta densidade, como os ossos do crânio (Fig.3C). A visibilização de estruturas localizadas na fossa caudal pode ficar prejudicada por causa de um artefato que ocorre geralmente nessa região denominado de endurecimento do feixe (Fig. $3 \mathrm{C})$. Esse é causado à medida que componentes de baixa energia do feixe de raios $X$ vão sendo absorvidos e o feixe se torna mais penetrante, resultando em áreas escuras (baixos valores de unidade Housfield). Ocorre geralmente em interfaces de alto contraste, como a região densa da parte petrosa do osso temporal e o tecido encefálico (Hathcock e Stickle, 1993; Tidwell e Jones, 1999).

Foram obtidas nesse estudo imagens em planos transversal e dorsal. Equipamentos que possuem recursos computacionais permitem a reconstrução em outros planos e a formação de imagens tridimensionais (Hathcock e Stickle, 1993; Tidwell e Jones, 1999). As imagens reconstruídas em outros planos não apresentam a mesma qualidade das imagens obtidas originalmente, embora nos equipamentos de última geração essa diferença na qualidade da imagem seja menos significativa. Além disso, a possibilidade de reconstrução diminui consideravelmente o tempo de aquisição das imagens e também evita que o paciente seja reposicionado durante o exame. Quando comparados os dois planos, transversal e dorsal, a localização de certas estruturas, como os ventrículos laterais, o corpo caloso, a região do quiasma óptico e o diencéfalo, foi melhor nos cortes transversais. Dessa forma, protocolos de aquisição de imagens tomográficas do encéfalo são realizados em cortes transversais. O encéfalo normal pela TC aparece como um tecido relativamente homogêneo. As doenças que podem causar alterações nas características físicas do tecido encefálico, diminuindo ou aumentando sua densidade, seja pela presença de massas, edema, áreas císticas, hemorrágicas ou focos de calcificação e, consequentemente, alterar a capacidade de atenuação do feixe de raios $\mathrm{X}$ em relação ao tecido normal, podem ser detectadas pelo exame tomográfico, como mostram diversos trabalhos na literatura (Tucker e Gavin, 1996; Snyder et al., 2006; MacKillop et al., 2007).

\section{CONCLUSÕES}

A tomografia é uma ferramenta diagnóstica importante para investigação do conteúdo craniano, sendo possível observar várias estruturas anatômicas encefálicas de forma direta ou relacionando-as com estruturas vizinhas que auxiliam na sua identificação.

\section{AGRADECIMENTOS}

Ao Prof. Dr. Franklin de Almeida Sterman (in memorium), pela orientação neste trabalho.

\section{REFERÊNCIAS}

ASSHEUER, J.; SAGER, M. Mri and ct atlas of the dog. Oxford: Blackwell Science, 1997. 482p.

BEITZ, A.J.; FLETCHER, T.F. The brain. In: EVANS, H.E. Anatomy of the dog. Philadelphia: W. B. Saunders, 1993. p.894-946.

COUTURIER, L.; DEGUEURCE, C.; RUEL, Y. et al. Anatomical study of cranial nerve emergence and skull foramina in the dog using magnetic resonance imaging and computed tomography. Vet. Radiol. Ultras., v.46, p.375383, 2005. 
DENNLER, M.; LANGE, E.M.; SCHMIED, O.; KASER-HOTZ, B. Imaging diagnosis: metastatic hemangiosarcoma causing cerebral hemorrhage in dog. Vet. Radiol. Ultras., v.48, p.138-140, 2007.

DYCE, K.M.; SACK, W.O.O. Sistema nervoso. In:__. Tratado de anatomia veterinária. Rio de Janeiro: Elsevier, 2004. p.256-317.

EVANS, H. E. The skeleton. In: Anatomy of the dog. Philadelphia: W. B. Saunders, 1993. p. 122-218.

FEENEY, D.A.; FLETCHER, T.F.; HARDY, R.M. Atlas of correlative imaging anatomy of the normal dog - ultrasound and computed tomography. Philadelphia: W. B. Saunders, 1991. 383p.

FIKE, J.R.; DRUY, E.M.; ZOOK, B.C. et al. Canine Anatomy as assessed by computerized tomography. Am. J. Vet. Resea., v.41, p.1823$1832,1980$.

FIKE, J.R.; LECOUNTER, R.A.; CANN, C.E. Anatomy of the canine brain using high resolution computed tomography. Vet. Radiol., v.22, p.236-243, 1981.

HAGE, M.C.F.N.S. Imagem por ressonância magnética na investigação da cabeça de cães. 2005. 139 f. Tese (Doutorado em Medicina Veterinária) - Faculdade de Medicina Veterinária e Zootecnia, Universidade de São Paulo, São Paulo.

HATHCOCH, J.T.; STICKLE, R.L. Principles and concepts of CT. Vet. Clin. of North Am.: Small Anim. Pract., v.23, p.399-415, 1993.

JEFFERY, N.D.; THAKKAR, C.H.; YARROW, T.G. Introduction of computed tomography of the canine brain. J. Sm. Anim. Pract., v.33, p.210, 1992.
KÖNIG, H.E.; LIEBICH, H.G.; CERVENY, C. Sistema Nervoso. In: KÖNIG, H.E.; LIEBICH, H.G. Anatomia dos animais domésticos. Porto Alegre: Artmed, 2004. p.203-275.

LODEN, D.; NORTON, F.; WOLFLA, L.; FORD, R.B. Diagnosis of intracranial lesions by computerized tomography in three dogs. J. Am. Anim. Hosp. Assoc., v.19, p. 303-308, 1983.

MACKILLOP, E.; THRALL, D.E.; RANCK, R.S. et al. Imaging diagnosis - synchronous primary brain tumors in a dog. Vet. Radiol. Ultras., v.48, p.550-553, 2007.

PHELPS, M.E; HOFFMAN, E.J.; TERPOGOSSIAN, M.M. Attenuation coefficients of various body tissues, fluids and lesions at photon energies of 18 to $36 \mathrm{keV}$. Radiology, v.117, p.573-583, 1975.

SCHALLER, O. Nomenclatura anatômica veterinária ilustrada. São Paulo: Editora Manole, 1999. 614p.

SNYDER, J.M.; SHOFER, F.S.; WINKLE, T.J.V.; MASSICOTTE, C. Canine intracranial primary neoplasia: 173 cases (1986-2003). J. Vet. Int. Med., v.20, p.669-675, 2006.

THOMAS, W.B. Nonneoplastic Disorders of the Brain. Clin.Tech.Small Anim. Pract., v.14, p.125147, 1999.

TIDWELL, A.S.; JONES, J.C. Advanced imaging concepts: a pictorial glossary of CT and MRI technology. Clin. Tech. in Small Anim. Pract., v.14, p.65-111, 1999.

TUCKER, R.L.; GAVIN, P.R. Brain Imaging. Vet. Clin. North Am.: Small Anim. Pract., v.26, p.735-757, 1996. 